\title{
Building Generation Expansion Plans - a Decision Aid Approach to use in Competitive Electricity Markets
}

\author{
Adelino J. C. Pereira, student member IEEE, João Tomé Saraiva, member IEEE
}

\begin{abstract}
Generation expansion planning gained a new dimension with the advent of electricity markets. It is now an activity decoupled from transmission and there are several agents competing to generate electricity and aiming at maximizing their individual profits. In view of this, it becomes more important to develop tools to help generation agents to build their expansion plans, internalizing several uncertainties in the model, an being able to simulate different possible reactions of the other competitors, given their impact in the profits of the agent being modelled. In this paper, we present a long-term decision aid tool that uses System Dynamics to model the long run of electricity markets together with Genetic Algorithms to solve the individual expansion problem of generation agents given their mixed-integer nature. Apart from the detailed description of the developed approach, the paper also includes a Case Study based on a four generation agent system to illustrate its application.
\end{abstract}

Index Terms-- generation expansion planning, competitive markets, long-term strategies, System Dynamics.

\section{INTRODUCTION}

$\mathrm{G}$ ENERATION Expansion Planning, GEP, is not a novel problem in the power systems area. Traditionally, it aimed at identifying the most adequate set of generators, including technologies and installed capacity, together with their sitting and commissioning along an extended planning period. This identification was typically driven by a cost objective function reflecting both investment and expected operation costs and considering a number of constraints. These included meeting the forecasted demand in each year of the planning horizon, ensuring that a specified value of a reliability index was not exceeded (as for instance the Loss of Load Expectancy), ensuring that the expansion plan was adequately diversified in order to cope with maximum values of installed capacities per technology and also ensuring that constraints expressing financial limitations were met. This was a complex problem given the involved long term planning horizon, the uncertainties affecting several parameters and data (as the demand level and the oil price) and also given its discrete nature arising from the limited number of expansion possibilities typically available for each technology. In any case, prior to the advent of electricity markets this long-term planning exercise was conducted in an integrated way together with the transmission expansion planning exercise in the scope of vertically integrated companies.

Adelino Coelho Pereira is with the Instituto Superior de Engenharia de Coimbra, Instituto Politécnico de Coimbra, Rua Pedro Nunes, 3030-199 Coimbra, Portugal, ajcp@isec.pt.

J. T. Saraiva is with Faculty of Engineering of Porto University and with the Power Systems Unit of INESC Porto, Rua Dr. Roberto Frias, 378, 4200465, Porto, Portugal, jsaraiva@fe.up.pt.
With the introduction of market mechanisms in the electricity sector, the GEP problem gained new dimensions and a renewed interest. This renewed interest is justified because the restructuring of power systems typically decoupled generation in several competing companies and unbundled generation from transmission. On the other hand, the GEP problem lost its integrated nature in the sense there isn't now a single national integrated expansion plan for the generation system, except if understood as a generic reference guideline prepared by some state agency. In this sense, each generation agent will now build its own plan aiming at maximizing the expected profits along the planning horizon resulting from selling electricity in the market and from the incurred investment, maintenance and operation costs. This problem is now much more complex than in the past because it is contaminated by uncertainties affecting the demand, the fuel prices, the electricity market prices and the investment and maintenance costs. Finally, the investment strategy developed by a particular agent is also affected and will have impacts from the strategies built or developed by other competitors.

In this paper we detail an approach to the GEP problem to aid each investor to build its own long-term plan, getting insight on the possible impacts due to the behavior of input parameters and, as a result, helping generation companies to build more robust plans. The approach aims at maximizing the profit of each generation agent, it builds a plan based on a number of candidate technologies and possible installed capacities and it models demand evolution and electricity price uncertainties using pdf functions. The resulting problem has discrete nature, which explains the adoption of Genetic Algorithms to solve it and the behavior of the market is modeled using System Dynamics as a very adequate tool to model long-term behaviors and interactions between different parameters and variables.

According to these ideas this paper is structured as follows. After this introductory Section, Section II details several approaches described in the literature regarding the GEP problem. Section III presents the mathematical formulation of the problem and Section IV details the developed solution algorithm. Section V describes a Case Study in which one generation agent is preparing its expansion plan in the presence of three other generation companies. The Case Study enumerates the assumptions, the data, both deterministic and modelled by pdf distributions and presents the expansion plan, the evolution of electricity prices, of the reserve margin and of LOLE. Finally, Section VI draws the most relevant conclusions of this work. 


\section{THE GEP PROBLEM IN THE LITERATURE}

Generation expansion planning, GEP, has been a matter of research in the scientific community as demonstrated by the number of publications on this topic. In this review we organized the references in three groups. The first one includes papers addressing the problem in terms of traditional vertically integrated companies. The second one includes papers addressing the GEP considering that the industry was liberalized and unbundled. The third one can be considered a subgroup of the previous set, in the sense that it corresponds to approaches using a particular tool, System Dynamics, to model the long-term behavior of electricity markets.

From a traditional point of view, reference [1] describes the expansion planning problem considering that it aims at defining an investment schedule to construct both generation plants and transmission lines so that it meets the demand and it minimizes investment and operation costs while ensuring an adequate level of reliability. This approach is able to consider uncertainties affecting several parameters and the resulting problem is solved using stochastic programming. In [2] it is described another integrated approach to build a schedule both for generation and transmission investments. The objective of this approach is to minimize the present value of investment and operation costs plus a penalty if there is unserved energy. The constraints include a representation of the transmission system based on the DC model and the problem is formulated as a Mixed Integer Linear Programming problem. Given the complexity of the GEP and its multiyear nature, [3, 4] detail the use of Benders Decomposition. For instance, in [4] the GEP is decomposed in a master problem that deals with the mixed integer investment problem together with sub-problems dealing with the optimal level of generation in each time period. These linear subproblems are used to generate Benders cuts to include in the master problem in the next iteration. The master problem is then solved using Genetic Algorithms. References [5, 6] detail multiobjective approaches to the GEP problem considering investment, operation and maintenance costs and environmental impacts (land use, accidents, the impact on ecosystems and emissions). The solution approach detailed in [5] corresponds to an iterative algorithm in which non dominated solutions are presented to the Decision Maker that can afterwards refine some of them investigating more deeply some areas of the solution space. Recognizing the integer nature of several variables, the approach described in [7] uses a Genetic Algorithm to solve the GEP. The problem minimizes investment, operation and depreciation costs along the planning horizon and the paper describes the coding strategy, the fitness function and the adopted genetic operators. In this line of research, [8] compares the use of several metaheuristics to the GEP problem including simulated annealing, genetic algorithms, particle swarm optimization and ant colonies. The authors advocate the use of AI-based heuristic techniques given they have large potential to solve complex planning and investment problems. Reference [9] also reviews the use emergent techniques to the GEP including expert systems, fuzzy logic, simulated annealing, genetic algorithms, hierarchy processes and decomposition methods.
Finally, [10] illustrates the use of simulated annealing and genetic algorithms to the GEP problem of Turkey.

Regarding the second group of papers, references [11 - 12] address general issues arising with the advent of liberalization and deregulation in the industry. As an example, reference [11] indicates that the advent of liberalization and deregulation brings more uncertainties, more price fluctuations namely in daily short term markets, there is a larger number of agents and the demand to be supplied by each individual agent is more difficult to predict. All these characteristics turn long-term traditional optimization techniques difficult to use because agents now prefer short-term decisions or the definition of flexible long-term strategies. Reference [13] addresses the impact of liberalization on investment long-term decisions. Several markets and geographical areas were investigated and the authors concluded that till 2003 there was on going enough investments in new generation assets except in the case of California, but there were several challenges to be addressed. The authors stress that governments were concerned with the adequacy of the generation systems and also with the composition of the investment mix in liberalized markets. References [14 - 16] describe different approaches to the GEP problem under competitive conditions. In [14] it is presented a model in which traditional generation utilities have to buy electricity from IPP's and the individual objective of each utility is to maximize its own profit admitting a specified level of selling electricity tariffs. Reference [15] describes an approach that admits several competing generation agents that are responsible for preparing their own expansion plans in view of input prices along the planning horizon. These individual plans are then submitted to an Upper Organization Level that checks the quality of the global plan evaluating the reserve margin and LOLP along the horizon. In this paper, the authors argue that the mentioned Upper Organization Level can correspond to an ISO but one doubts if this doesn't still correspond to a too centralized view in the sense that in a market system agents would hardly accept turning their plans public. Finally, in [16] it is described an approach in which the market is represented by linear supply and demand functions and stochastic dynamic programming is used to solve the investment problem together with discrete Markov chains to model the uncertainty affecting the demand.

Finally, in recent years System Dynamics started to be used to model the long-term behavior of electricity markets. System Dynamics was conceived by J. W. Forrester still in the 1960's and [17] summarizes his contributions and its main concepts. References [18 - 20] illustrate the application of these concepts to the GEP problem. For instance, in $[19,20]$ it is detailed how the Dynamic Model is built together with the relations of the investment problem with the energy and capacity markets and with the economic environment so that it is possible to capture long-term dependencies. Reference [20] details the characteristics of investments in generation capacity, models the reaction of investors acting in electricity markets, models the supply and the demand side and the mechanism to form electricity prices. Given the increasing use of System Dynamics in several areas there are several software packages that facilitate the use of these concepts. References [21, 22] detail one of these commercial packages. 


\section{MATHEMATICAL FORMULATION}

\section{A. General aspects}

The developed approach formulates the GEP problem to aid generation companies to take decisions regarding investments on generation capacity. In a competitive environment there are several GENCO's competing to supply electricity leading to a more risky environment to develop new investments. In the developed approach, each GENCO maximizes its expected profit considering the behaviour of the competitors, the uncertainties associated to the evolution of electricity market prices, the financing scheme, the characteristics of the alternative stations (both from an economic and technical point of view), and the investment, operation and maintenance costs.

In order to build the expansion plans, each GENCO solves the problem detailed in Section III B. The long-term operation of the electricity market is modelled using System Dynamics as described in Section III C. This long-term market modelling aims at quantifying the evolution of electricity prices, of the capacity factors of generation stations of each technology, the total demand, the demand to be supplied by each technology, the impact of generation using renewables (namely hydro's and wind parks) and the impact of the decisions assumed by the other competing GENCO's along the horizon.

\section{B. Optimisation problem}

The GEP problem to be solved by each GENCO is formulated by $(1-6)$. The objective function maximizes the expected profit resulting from selling electricity in the market and from operation, maintenance and investment costs. This function also includes a revenue term associated with a payment for capacity declared in reserve as a way to improve the reliability of the system. As a result it is built an expansion plan quantifying how much it is invested in each technology and the commissioning period. Uncertainties affecting the electricity price, the capacity factors, the investment costs, the fix and variable operation and maintenance costs and the interest rate are modelled by fdp's. In order to extract values from these fdp's it is used the Monte Carlo Simulation method.

$$
\max z_{i}=\sum_{t=1}^{T}\left\{\sum_{j=1}^{M}\left[\begin{array}{l}
\sum_{n=1}^{t c j}\left(-\operatorname{Ccap}_{j} X_{t}^{i j}\right)+ \\
+\sum_{k=t+t c j}^{T u t+t c j}\left(\begin{array}{l}
\left(\pi^{t} \cdot X_{t}^{i j} \cdot \alpha_{t}^{i j}+\left(1-\alpha_{t}^{i j}\right) \cdot X_{t}^{i j} \cdot p_{t c a p k}\right)- \\
-C c a p_{k} \cdot X_{t}^{i j}- \\
-\left(C o p_{k}^{i j} \cdot X_{t}^{i j} \cdot \alpha_{t}^{i j}+C f i x_{k}^{i j} \cdot X_{k}^{i j}\right)
\end{array}\right)
\end{array}\right]\right\}
$$

$$
\begin{aligned}
\text { subj } & \sum_{t=1}^{T n} X_{t}^{i j} \leq \text { MCapI }_{T n}^{i, j} \\
& \sum_{t=1}^{T n} \sum_{j=1}^{M} X_{t}^{i j} \leq \operatorname{MCapI}_{T n}^{i} \\
& \text { A }_{\text {cum }}^{i}{ }_{t=0}^{i}+\sum_{t=1}^{T} \sum_{j=1}^{M} X_{t}^{i j} \leq \text { CAcum }_{\text {Total }}^{i} \\
& \sum_{t=1}^{T n} \sum_{j=1}^{M} X_{t}^{i j} \cdot \operatorname{Cinv}_{t}^{j} \leq \operatorname{CapDisp}_{T n}^{i} \\
& t=1, \ldots T ; j=1, \ldots, M ; \text { Tn }- \text { it takes values in }\{1, \ldots, T\}
\end{aligned}
$$

\begin{tabular}{|c|c|}
\hline$T$ & number of stages in the planning horizon; \\
\hline$t$ & stage in the planning horizon (year); \\
\hline Tut & $\begin{array}{l}\text { lifetime of each generation station depending } \\
\text { the technology; (year); }\end{array}$ \\
\hline$t c_{j}$ & $\begin{array}{l}\text { construction time of a station of technology } \mathrm{j} \\
\text { (year); }\end{array}$ \\
\hline$T n$ & extension of the planning horizon (year); \\
\hline$i$ & GENCO index; \\
\hline$M$ & number of candidate technologies; \\
\hline$j$ & index for candidate expansion technology; \\
\hline$\pi^{t}$ & price of electricity in year $t$ \\
\hline$\alpha_{t}^{i, j}$ & $\begin{array}{l}\text { capacity factor in year } t \text {, for GENCO } \mathrm{i} \text { and for } \\
\text { technology } j \text {; }\end{array}$ \\
\hline $\operatorname{Cinv}_{t}^{j}$ & investment cost for technology $j$ in year $t$; \\
\hline $\operatorname{Cop}_{t}^{j}$ & $\begin{array}{l}\text { variable operation and maintenance cost for } \\
\text { technology } j \text { in } t \text {; }\end{array}$ \\
\hline$p_{\text {tcapk }}$ & available capacity payment ( $€ / \mathrm{MW})$ \\
\hline Ccap $_{j}$ & $\begin{array}{l}\text { capital cost per installed MW due to the loan } \\
\text { (€/MW.year); }\end{array}$ \\
\hline$C f i x_{k}^{i j}$ & $\begin{array}{l}\text { fix operation cost for technology } \mathrm{j} \text {, in year } \mathrm{t} \\
\text { (€/MW.year); }\end{array}$ \\
\hline$X_{t}^{i, j}$ & $\begin{array}{l}\text { new capacity of technology } \mathrm{j} \text {, in year } \mathrm{t} \text { for } \\
\text { GENCO } \mathrm{i}_{i}\end{array}$ \\
\hline CAcum $_{t=0}^{i}$ & $\begin{array}{l}\text { installed capacity owned by GENCO i till the } \\
\text { planning period starts (MW); }\end{array}$ \\
\hline CAcum $_{\text {Total }}^{i}$ & $\begin{array}{l}\text { maximum value admitted for the installed } \\
\text { capacity owned by GENCO } \mathrm{i} \text { when the } \\
\text { planning horizon ends (MW); }\end{array}$ \\
\hline CapDisp ${ }_{T n}^{i}$ & $\begin{array}{l}\text { financial resources available to invest in year t } \\
\text { or in all } T n \text { periods, by GENCO i }(€) \text {; }\end{array}$ \\
\hline $\operatorname{MCapI}_{T n}^{i}$ & $\begin{array}{l}\text { maximum capacity that GENCO } \mathrm{i} \text { can install } \\
\text { in all } \mathrm{M} \text { technologies in year } \mathrm{t} \text { or in all } T n \\
\text { periods }(\mathrm{MW}) \text {; }\end{array}$ \\
\hline $\operatorname{MCapI}_{T n}^{i, j}$ & $\begin{array}{l}\text { maximum capacity that GENCO i can install } \\
\text { on tech. } \mathrm{j} \text { in year t or in all } T n \text { periods (MW). }\end{array}$ \\
\hline
\end{tabular}

In this formulation:

The revenues to be obtained by each GENCO along the lifetime of the generators are influenced by the capacity to install of each technology (decision variables), by the capacity factor of each technology and by the electricity prices. The capacity to install of each technology is also determined by the normalized available values. This issue is relevant because it means that problem $(1-6)$ has a combinatorial nature and it corresponds to a mixed integer problem.

The evolution of the electricity price to be used in $(1-6)$ is obtained by the long-term simulation of the electricity market using System Dynamics. This evolution is determined by the generation mix in each period and along the planning horizon, by the evolution of oil, coal and gas prices, by the installed capacity in renewables (namely hydros and wind parks) as well as by the output of these units. The generation mix is determined by the expansion plans of each GENCO which means that the decisions of all agents will influence the particular decisions of each of them.

Most of these factors are not directly controlled by each GENCO and so it is important to internalise uncertainties. In this approach we used Log-Normal fdp's to model the electricity price in each year. The mean value comes from the System Dynamic model and the standard deviation was set at 
$10 \%$ of the mean. The capacity factor is also affected by uncertainty and so we used for each technology a Normal fdp. Its mean value comes from the System Dynamic model and the standard deviation was set at $5 \%$ of the mean.

Regarding the cost of capital resulting from the investment in each station, it corresponds to the second term in (1). This cost depends on the cost per MW of each technology, interest rate and amortization period. Its value was obtained using (7).

$$
\operatorname{Ccap}_{j}=\frac{\text { Constr }_{j}}{\sum_{k=1}^{\text {Tamort }} \frac{1}{\left(1+\frac{\text { tact }_{k}}{100}\right)^{k}}}
$$

In this expression:

$\begin{array}{ll}\text { Constr }_{j} & \begin{array}{l}\text { construction cost per MW of technology j } \\ (€ / \mathrm{MW}) ;\end{array} \\ \text { tact }_{k} & \text { interest rate specified for period k (\%/year); } \\ \text { Tamort } & \text { amortization period (year); } \\ \text { Ccap }_{j} & \text { cost of capital per installed MW (€/MW.year); }\end{array}$

Regarding the constraints, (2) limits the installed capacity of technology $\mathrm{j}$ and it can be applied to individual periods or for the entire Tn periods. This limit can be established by each GENCO or can be set by the regulatory agency or can result from global objectives of energy policy. This means controlling the share of a technology $\mathrm{j}$ in the generation mix of a entire country or of individual GENCO's.

Constraint (3) limits the capacity that GENCO $i$ will install for all $M$ candidate technologies. Once again, this limit can be established for individual periods, or for all Tn periods. Constraint (4) establishes a limit for the entire capacity operated by GENCO $i$ considering both the capacity installed prior to this expansion exercise and the investments of the plan being built. This limit can be set by regulatory authorities as an attempt to limit the share of each generation agent in the generation mix, namely to prevent market power that could lead to the distortion of competition in the electricity market.

Constraint (5) models the financial constraints felt by each GENCO to invest in a particular year t or in all Tn years. This limit is established by each GENCO according to its financial strength and we admitted that the required capital is available in the beginning of the construction period of a new unit.

Given the discrete nature of the problem $(1-6)$ we used Genetic Algorithms together with the Monte Carlo simulation method to build an algorithm that adequately addresses all the characteristics of this problem. In this scope, GENCO's prepare individual expansion plans to maximize their profit and then these plans are evaluated from an adequacy global point of view namely to check the reserve margin and the Loss of Load Expectancy, LOLE, along the planning horizon. This means that the yearly values obtained for the reserve margin and for LOLE are compared with maximum values. In a similar way, the total installed capacity per technology is also compared against limit values. Regarding the LOLE, it is important to notice that the Grid Code of several countries establish maximum values for this adequacy index expressed in terms of the maximum number of hours during which the demand may not be attended.
If necessary, that is if the investments plans are unable to obtain an adequate adequacy, we can define incentive schemes to induce new investments, for instance simulating an increase of the capacity payment per MW in reserve. These alterations are then conveyed to the investors so that the expansion plans are updated as detailed in [23].

\section{Long Term Modelling using System Dynamics}

System Dynamics was used to simulate the long-term behaviour of electricity markets. The solution of this problem outputs important results used by each GENCO in their individual optimisation problems. These outputs are the evolution of the demand, of electricity prices, capacity factors of each technology, impact of installed capacity in each technology and decommissioning of existing units.

In an initial step, the individual expansion plans obtained by the GENCO's are used to simulate the operation of the electricity market. The results obtained are then used by the GENCOs to update their plans. If there is any change on the individual plans, this information is used to run the Dynamic Model again and the results are sent back to the GENCO's. This process corresponds to a feedback loop over the most relevant parameters to be used by the GENCO's when building their plans and allows internalising the impact of the decisions of the other competitors.

Following [20], Figure 1 presents the generic casual diagram to model the interrelations between the components relevant for the operation of the electricity market. According to this diagram, there is a positive cause-effect relation between the evolution of the installed capacity and the reserve and a negative impact on the available power from decommissioning capacity. It is also possible to notice that an increase in the demand originates a reduction of the reserve and this reduction leads to an increase of the electricity price.

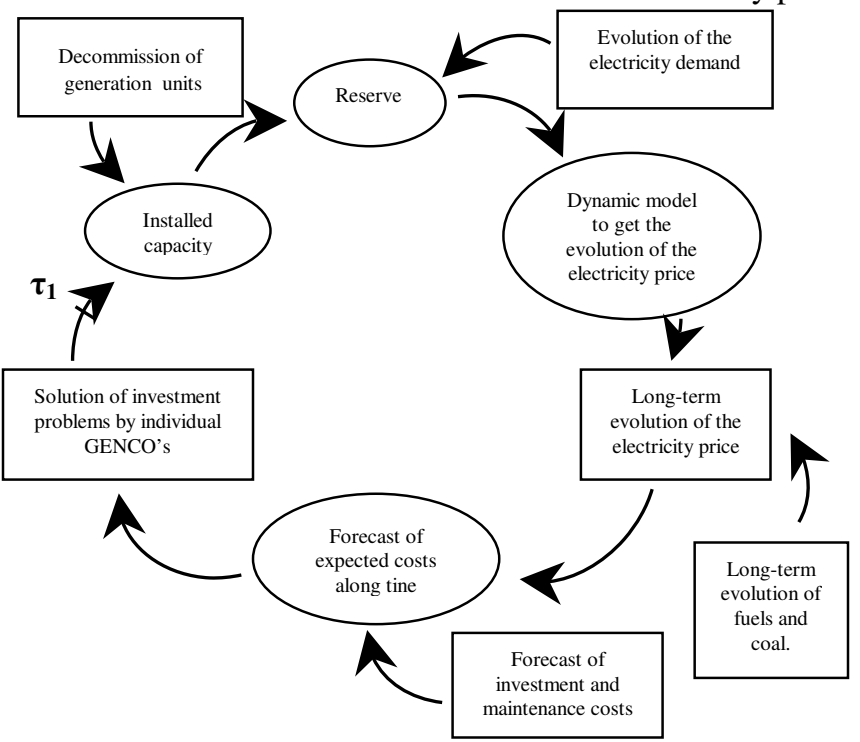

Fig. 1. Casual diagram representing the interactions in the electricity market.

This diagram also includes the delays that can have impacts on the behaviour of the market. As an example, there is a delay between the moment in which an investment decision is taken and the moment in which this new capacity is available. This delay corresponds to the construction and commission time. 
Considering this diagram together with the optimization problem $(1-6)$ to be solved by each GENCO, it is possible to conclude that there is a feedback loop in which the individual investment decisions (outputs of problem $(1-6)$ ) determine the evolution of the installed capacity that determines the behaviour of the reserve. Considering the existing generation mix, it is then obtained the long-term evolution of the electricity price certainly affected by the evolution of the price of fuels. Electricity prices together with the evolution of fix and variable costs will finally determine investment decisions.

In this approach we considered three sets of power stations - hydro stations (run-of-river and reservoirs), thermal stations (using coal, fuel and natural gas) and renewables, namely wind parks. In [24] we described the main characteristics of the Dynamic Model of the electricity sector. This model integrates three main sub-models to simulate the annual electricity demand, to simulate the supply of the demand by the different available technologies and to obtain the evolution of the electricity market prices. The annual electricity demand is influenced by the demand rate evolution, by the reference demand, by the electricity price in the beginning of the planning period and by its evolution and by the elasticity demand/price. The demand rate is modelled by a stochastic variable in order to incorporate the associated uncertainty and we used a Mean Regression Process to represent it.

The simulation of the supply considers the mentioned three sets of stations and the commissioning of new ones. Regarding wind parks and hydro stations we use historical series that reflect both the availability of the power station and the availability of the natural resource together with the installed capacity. This means we used fdp's to consider this uncertainty and a stochastic process to extract values from it. For wind parks the mean value of the capacity factor varies from 0.20 to 0.30. For hydro stations we admitted three possible intervals that can be used to model an average hydro year (capacity factor ranging from 0.26 to 0.30 ), dry year (capacity factor from 0,20 to 0,25 ) and wet year (capacity factor from 0.31 to 0.37). The Dynamic Model selects in a random way the type of year.

Regarding the thermal stations, the Dynamic Model obtains the evolution of the capacity factor of each technology along the planning horizon. In order to this, for each technology we use a function that relates the marginal generation cost with the capacity factor, as it is illustrated in Figure 2 admitting that the different steps were normalized.

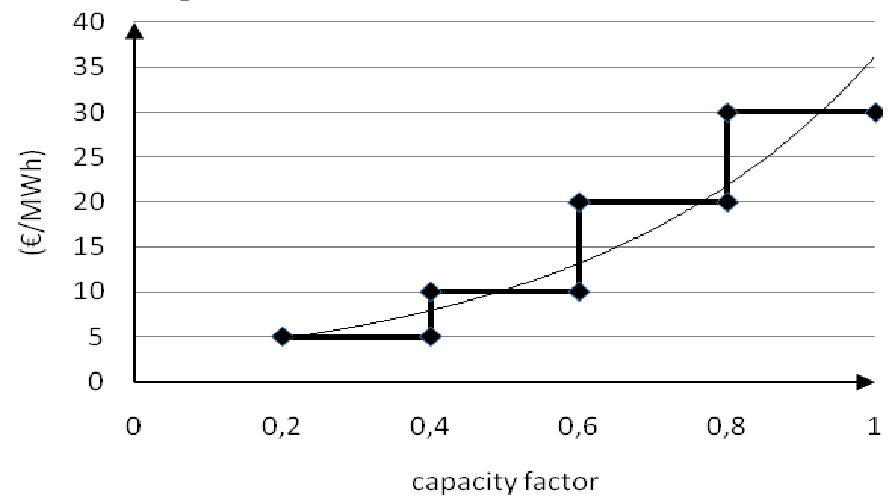

Fig. 2. Normalized marginal generation cost function for a thermal station.
The output of each station depends on the installed capacity (MW), on the availability along the year (h/year) and on the electricity market price and it influences the capacity factor of each technology. The availability of each station depends on the duration of the maintenance actions according to the technology. In order to adequately model this issue, the planning horizon can be discretized in hours, weeks, or months. The Dynamic Model will then provide the output in each of the periods of the discretized horizon.

The sub model developed to simulate the evolution of the electricity market price uses the output of the simulation of the supply and of the electricity demand. We then obtain the evolution of electricity prices considering a reference price in the beginning of the horizon. The evolution of the price along time is influenced by the demand and generation evolutions. The price is obtained for each period according to the selected discretization step (hour, day or week) and the application can then compute monthly or yearly average prices.

\section{DEVEloped SOlution Algorithm}

Figure 3 presents the generic structure of the algorithm used in the developed application to help generation agents to take robust investment decisions regarding new capacity. This figure also highlights the relations with other sub models as the evaluation of the set of plans developed by the GENCO's (computation of reserve margin and LOLE) as well as the optimization problems to be solved by each GENCO.

As detailed above, the Dynamic Model simulates the longterm behaviour of the electricity market and outputs the evolution of the electricity prices, of the capacity factor of each technology and of the demand (depending on the generation mix and its evolution according to the individual plans developed by each generation agent).

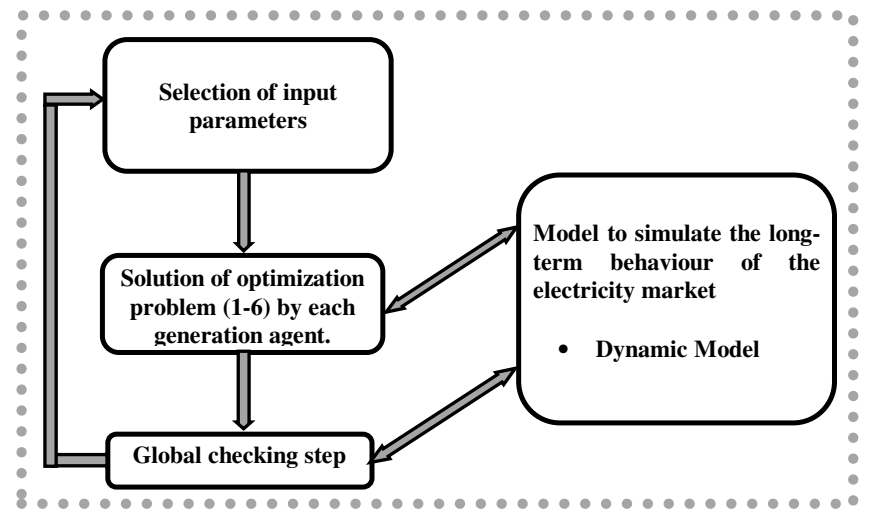

Fig. 3. Generic algorithm of the developed application.

In the first step, the GENCO's solve the problem (1 - 6) considering the information characterizing each available technology, its investment strategy and other constraints detailed in Section III.B. In this initial step each GENCO uses an estimate of the evolution of the electricity price, capacity factors and demand as well as for the generation of hydro stations and wind parks. The investment plans are then used by the Dynamic Model to refine the evolution of the electricity price, of the capacity factors and of the demand and are also checked from a global point of view computing the reserve 
margin and the LOLE. This information is conveyed to the GENCO's so that they individually solve a new problem (1 $6)$. This iterative scheme is repeated until there are no changes on the developed investment plans in two successive iterations. The final result corresponds to the investment plan of each GENCO, including the selected technologies, the capacities and the commissioning timing. In a supplementary way, the application provides the evolution of the electricity prices, of the capacity factors of each technology, the reserve level and the value of LOLE for each period of the planning horizon.

\section{CASE STUDY}

\section{A. Data and Assumptions}

In this section we will detail the results obtained using the application described in Sections III and IV to a Case Study in which we considered a generation system with installed capacity of $7000 \mathrm{MW}$ in the beginning of the planning period. Table I details the characteristics of the existing technologies regarding the rated power, the fix operation and maintenance cost, the variable cost and the Forced Outage Rate, FOR. Regarding the thermal stations, we used curves relating the marginal operation cost with the capacity factor. The capacity factors were grouped in the following four intervals: $[0.2 ; 0.4]$; $10.4 ; 0.6] ; 10.6 ; 0.8]$ e $] 0.8 ; 1]$. Then, for each of these intervals we specified the corresponding marginal operation cost as indicated in the fifth column of Table I so that we finally built curves similar to the one in Figure 2.

Table I - Characteristics of the existing technologies.

\begin{tabular}{|c|c|c|c|c|c|}
\hline $\begin{array}{c}\text { no. } \\
\text { Units }\end{array}$ & Technology & $\begin{array}{c}\text { Rated power } \\
(\mathrm{MW})\end{array}$ & $\begin{array}{c}\text { Fix operation and } \\
\text { maintenance cost } \\
(€ / \mathrm{MW} . \text { year })\end{array}$ & $\begin{array}{c}\text { Operation Cost } \\
(€ / \mathrm{MW} . \mathrm{h})\end{array}$ & FOR \\
\hline 4 & Coal_1 & 300 & 13000 & $12 ; 17 ; 30 ; 42$ & 0.02 \\
\hline 2 & Coal_2 & 400 & 12000 & $10 ; 22 ; 35 ; 45$ & 0.02 \\
\hline 4 & Gas turbine & 250 & 8500 & $15 ; 30 ; 43 ; 55$ & 0.01 \\
\hline 2 & Oil & 200 & 11000 & $20 ; 35 ; 45 ; 60$ & 0.03 \\
\hline 4 & CCGT & 400 & 7000 & $10 ; 20 ; 32 ; 45$ & 0.01 \\
\hline 5 & wind parks & 200 & - & & - \\
\hline 4 & hydro & 250 & - & & - \\
\hline
\end{tabular}

Regarding this initial generation mix, we admitted that the ownership of each generation agent follows the shares indicated below:

- GENCO_1 - 25\% of the installed capacity;

- GENCO_2 - 15\% of the installed capacity;

- GENCO_3 - 20\% of the installed capacity;

- GENCO_4 - 20\% of the installed capacity;

- other GENCO's - $20 \%$ of the installed capacity.

Table II details the characteristics of the 4 candidate technologies regarding the available capacities, the investment cost, the fix operation and maintenance cost, the cost of capital and the FOR. The variable operation costs were defined in four steps as explained above for the thermal stations in Table I. In this case, for the 4 candidate technologies we used:

- Tech_1 - 12, 30, 45 and 50 €/MW.h;

- Tech_2 - 10, 22, 33 and 45 €/MW.h;

- Tech_3 - 10, 18, 30 and 40 €/MW.h;

- Tech_4 - 8, 15, 25 and 35 €/MW.h.
Table II - Characterization of the 4 alternative technologies to install.

\begin{tabular}{|c|c|c|c|c|c|}
\hline $\begin{array}{c}\text { Type of } \\
\text { technology }\end{array}$ & $\begin{array}{c}\text { Available } \\
\text { capacities (MW) }\end{array}$ & $\begin{array}{c}\text { Investment } \\
\text { cost }(€ / \mathrm{MW})\end{array}$ & $\begin{array}{c}\text { Fix operation and } \\
\text { maintenance cost } \\
(€ / \mathrm{MW} . y e a r)\end{array}$ & $\begin{array}{c}\text { Cost of capital } \\
\text { with the loan } \\
(€ / \mathrm{MW} . y e a r)\end{array}$ & FOR \\
\hline Tech_1 & $\begin{array}{c}100 \text { or } 150 \text { or } \\
200\end{array}$ & 450000 & 6000 & 42230.40 & 0.02 \\
\hline Tech_2 & 100 or 200 & 550000 & 6137 & 44133.40 & 0.02 \\
\hline Tech_3 & $\begin{array}{r}100 \text { or } 200 \text { or } \\
250\end{array}$ & 800000 & 7655 & 49666.72 & 0.01 \\
\hline Tech_4 & 200 or 300 & 1000000 & 12482 & 65051.44 & 0.01 \\
\hline
\end{tabular}

The construction period and lifetime of the stations of each technology are of 2 and 25 years for Tech_1 and Tech_2 and 3 and 30 years for Tech_3 and Tech_4. The planning horizon corresponds to 15 years and during this horizon we admitted that the maximum value of LOLE is 3 hour/year and the reserve margin should be in the range [20\%; 35\%]. During this horizon we also admitted that they will be commissioned:

- 200 MW of capacity in wind parks in year 4;

- $250 \mathrm{MW}$ of capacity in wind parks in year 6;

- $400 \mathrm{MW}$ of capacity in wind parks in year 11 ;

- $200 \mathrm{MW}$ of hydro in year 3;

- $150 \mathrm{MW}$ of hydro in year 7 .

When running the investment optimisation problems we considered the following financial constraints:

- GENCO_1 - $250 \mathrm{M} €$ available in the first 5 years, 200 $\mathrm{M} €$ from the $6^{\text {th }}$ to the $10^{\text {th }}$ year and $100 \mathrm{M} €$ from the $11^{\text {th }}$ till the $15^{\text {th }}$ year;

- GENCO_2 - $300 \mathrm{M} €$ available in the first 5 years, 400 M€ from the $6^{\text {th }}$ to the $10^{\text {th }}$ year and $200 \mathrm{M} €$ from the $11^{\text {th }}$ till the $15^{\text {th }}$ year;

- GENCO_3 - $600 \mathrm{M} €$ available in the first 5 years, 500 $\mathrm{M} €$ from the $6^{\text {th }}$ to the $10^{\text {th }}$ year and $500 \mathrm{M} €$ from the $11^{\text {th }}$ till the $15^{\text {th }}$ year;

- GENCO_4 - $600 \mathrm{M} €$ available in the first 5 years, 250 $\mathrm{M} €$ from the $6^{\text {th }}$ to the $10^{\text {th }}$ year and $600 \mathrm{M} €$ from the $11^{\text {th }}$ till the $15^{\text {th }}$ year.

We also admitted that GENCO_1 is interested to invest in all the four candidate technologies, GENCO_2 is interested to invest in Tech_1 and Tech_2, and GENCOs 3 and 4 are only interested in investing in Tech_3 and Tech_4. Due to regulatory settings, we also admitted that the share of each GENCO regarding the installed capacity can not exceed $35 \%$ of the total. The share of each GENCO regarding new capacity will range between 15 and $35 \%$.

Finally, Table III presents the values of the parameters used to run the dynamic simulation of the electricity market. It includes the values of the initial demand growth rate, the longrun demand growth rate, the speed of reversion, the volatility of the process, the reference electricity demand in the initial year $t=0$, the demand/price elasticity, the duration of the planning horizon and the price of electricity in initial year $t=0$.

Table III - Data for System Dynamic simulation.

\begin{tabular}{|l|c|l|c|}
\hline$t_{0}(\% /$ year $)$ & 3 & Dref $_{O}(\mathrm{GWh} /$ year $)$ & 40000 \\
\hline$t_{L P}(\% /$ year $)$ & 4 & $E_{D P}$ & 0.25 \\
\hline$\eta$ & 0.50 & $T$ (years) & 15 \\
\hline$\delta$ (\%/year) & 0.40 & $\pi^{t 0}(€ / \mathrm{MWh})$ & 35 \\
\hline
\end{tabular}




\section{B. Results Expansion Plans}

Figures 4 to 7 present the final expansion plans obtained for the four GENCO's. These results are influenced by the constraints associated to the activity of each GENCO and also by the global values imposed for LOLE, for the reserve margin and for the shares of each GENCO on the total and on the new installed capacity. During the solution of the investment planning problem, the constraints associated with the limits of the installed capacity of each technology, the maximum share of each GENCO in the installed capacity in each period and the maximum admitted reserve margin were frequently violated. The results indicate that market conditions are attractive for new capacity investments, leading to large reserve margins along the horizon.

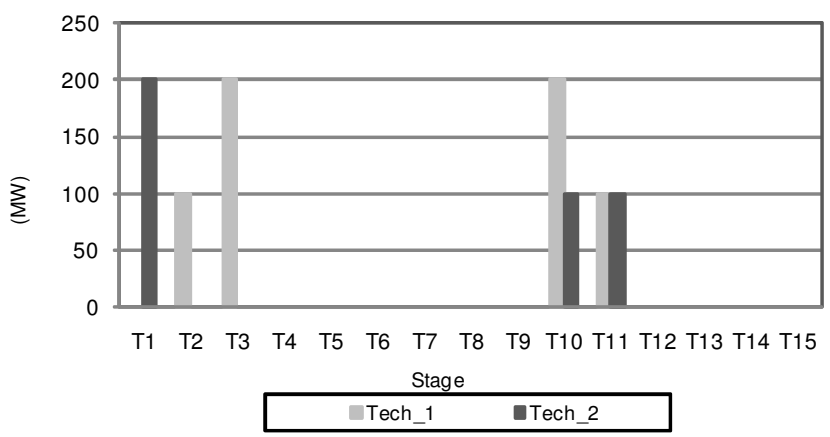

Fig. 4. Generation expansion plan obtained for Genco_1.

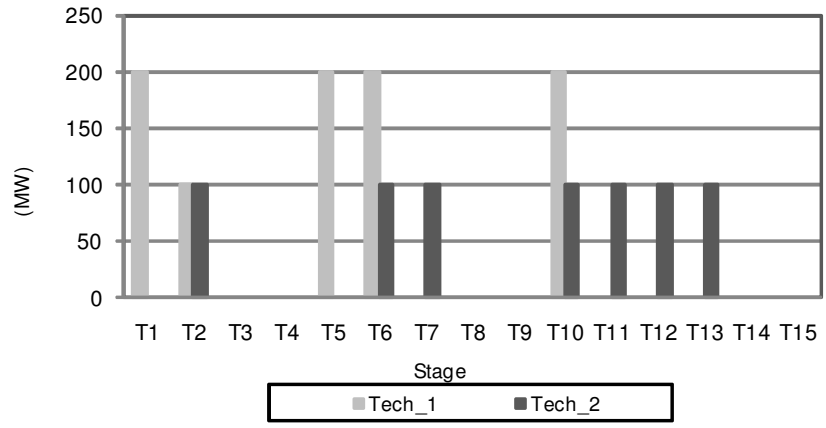

Fig. 5. Generation expansion plan obtained for Genco_2.

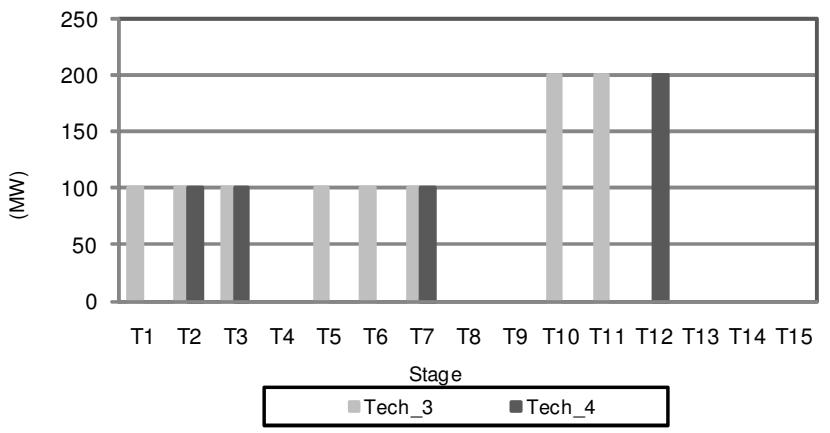

Fig. 6. Generation expansion plan obtained for Genco_3.

The share of each GENCO regarding the new capacity is as follows: $17.86 \%$ for GENCO_1, $28.58 \%$ for GENCO_2, $26.78 \%$ for GENCO_3 and $26.78 \%$ for GENCO_4. The distribution of the new capacity regarding the four technologies is as follows: $26.79 \%$ for Tech_1, $19.64 \%$ for Tech_2, $30.36 \%$ for Tech_3 and $23.21 \%$ for Tech_4.

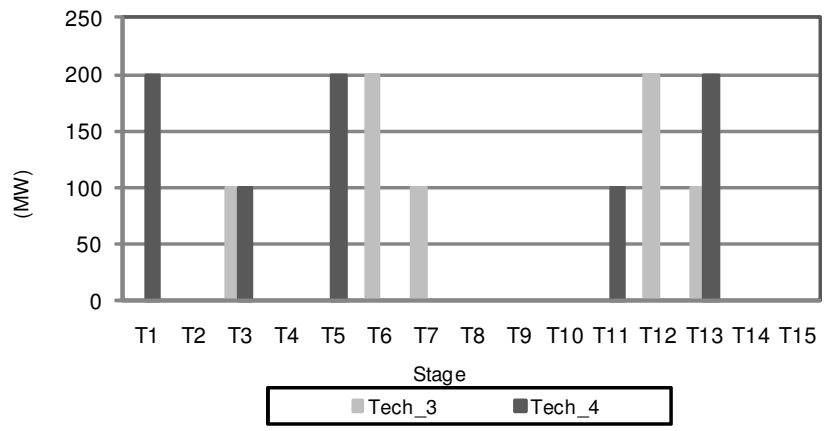

Fig. 7. Generation expansion plan obtained for Genco_4.

C. Evolution of the Demand, of the Electricity Price, of the Reserve Margin and of LOLE

Figure 8 presents the evolution of the annual demand along the 15 years as a result of the dynamic simulation of the market and Figure 9 presents the evolution of the average annual electricity market price. This evolution is influenced by the evolution of the generation mix as a result of the implementation of the investment plans, by the evolution of the demand as indicated in Figure 8 and also by the output of wind parks and hydro stations.

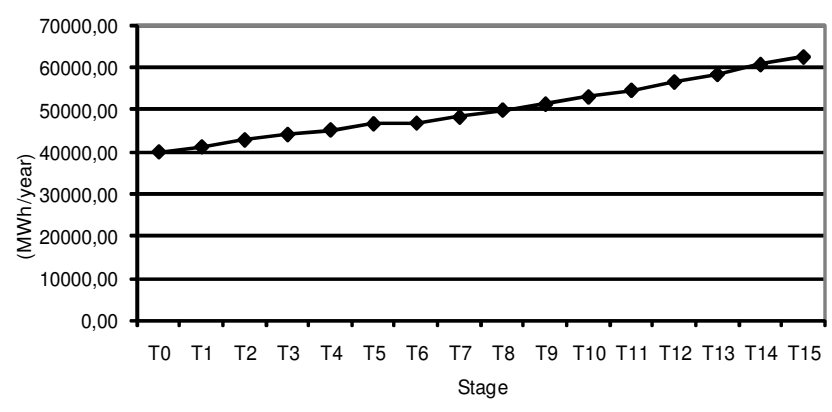

Fig. 8. Evolution of the demand along the planning horizon.

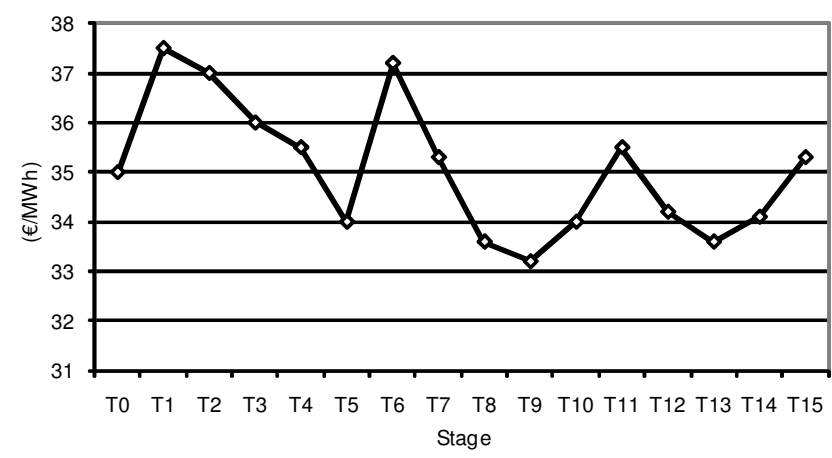

Fig. 9. Evolution of the electricity price along the planning horizon.

Figures 10 and 11 illustrate the evolution of the reserve margin and of LOLE. Regarding the reserve margin, this graph indicates that it is reached the maximum admitted value of $35 \%$ in some periods. In the remaining periods the reserve margin is close to $30 \%$. This shows that the expansion plans developed by the GENCO's are adequate to address the evolution of the demand. LOLE shows little variations along the 15 years and, in any case, always well below the limit of 3 $\mathrm{h}$ /year. Figure 11 also shows that when the reserve margin is lower, the value of LOLE tends to be larger. 


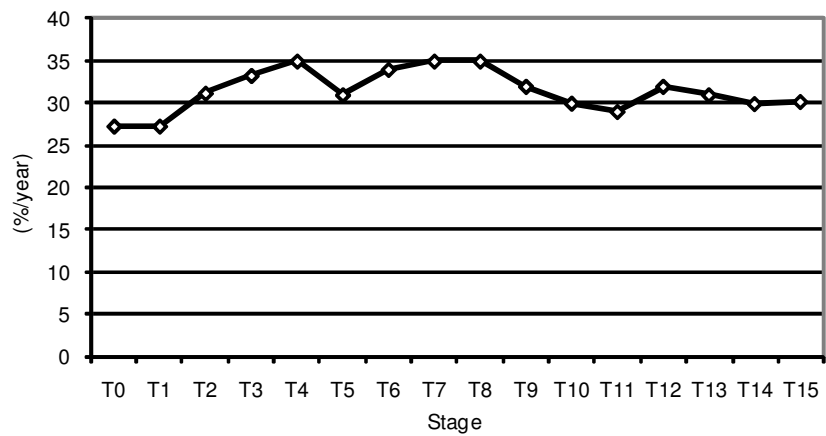

Fig. 10. Evolution of the reserve margin.

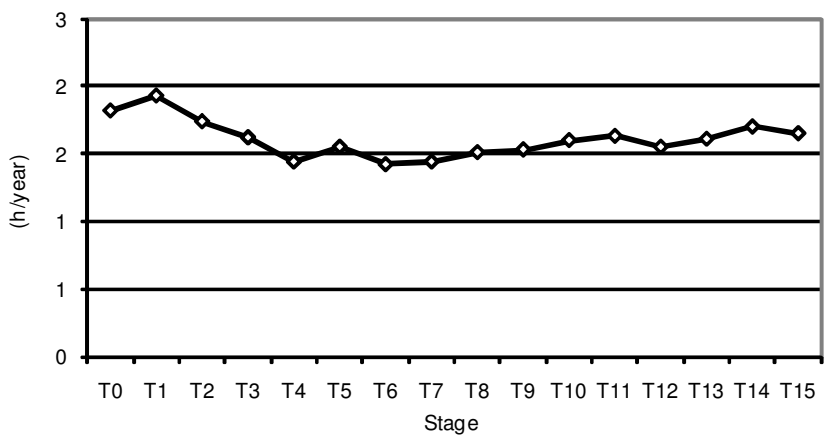

Fig. 11. Evolution of the LOLE.

\section{Sensitivity Analysis - Increase of the Cost of Fuel}

In order to evaluate the robustness of the developed plans, we conducted a sensitivity analysis considering that the cost of fuel used by Tech_3 was increased by $10 \%$ regarding the values used in the previous simulation, and indicated in Section V.A. This means that the steps of the curve relating the marginal operation costs with the capacity factor of Tech_3 have now the following values: 11, 19.8, 33 and $44 € / M W . h$.

After running the planning exercise again, it is possible to conclude that the expansion plans of GENCO_1 and of GENCO_2 do not change. Regarding the investment plans of GENCO_3 and of GENCO_4, it occurred a reduction of the investments in power stations of Tech_3. For Genco_3 this reduction was of $200 \mathrm{MW}$ and also of $200 \mathrm{MW}$ for GENCO_4. Figure 12 and 13 display the new plans obtained for these two GENCO's.

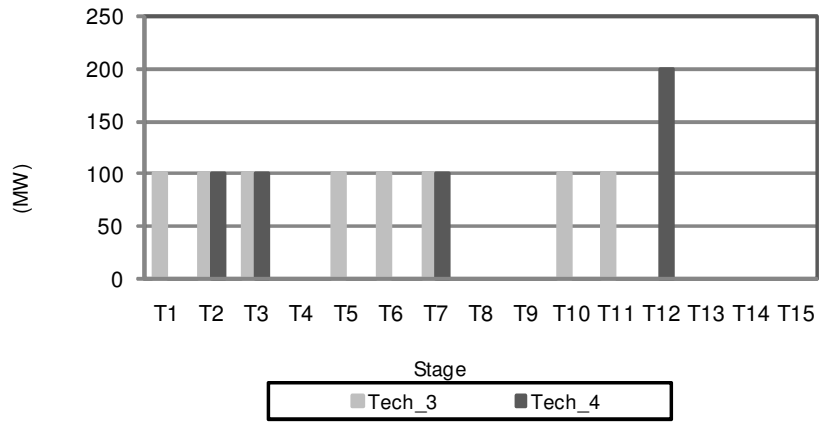

Fig. 12. New generation expansion plan obtained for Genco_3

The evolution of the other results didn't show significant changes regarding the values reported in Section V.C. This is due to the fact that the reserve margin continues to display values close to $30 \%$, which lies in the upper part of the admissible interval.

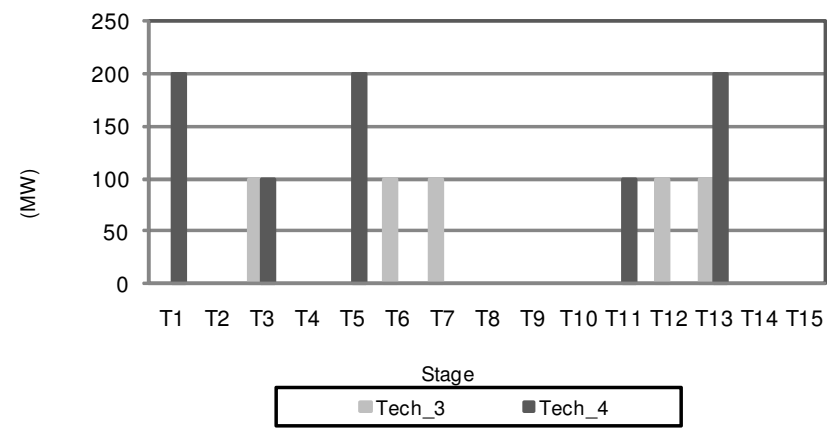

Fig. 13. New generation expansion plan obtained for Genco_4

\section{E. Sensitivity Analysis - Increase of the Investment Cost}

In a second step, we decided to investigate the impact of increasing the investment cost of Tech_1 by 20\% regarding the value indicated in Table II of Section V.A. This means that the investment cost is now $540000 € / \mathrm{MW}$ and $50676 € / \mathrm{MW}$.year for the cost of capital with the loan. As a result of this change, the investment plans of GENCO's 3 and 4 didn't change. The new plans obtained for GENCO_1 and for GENCO_2 are display in Figures 14 and 15.

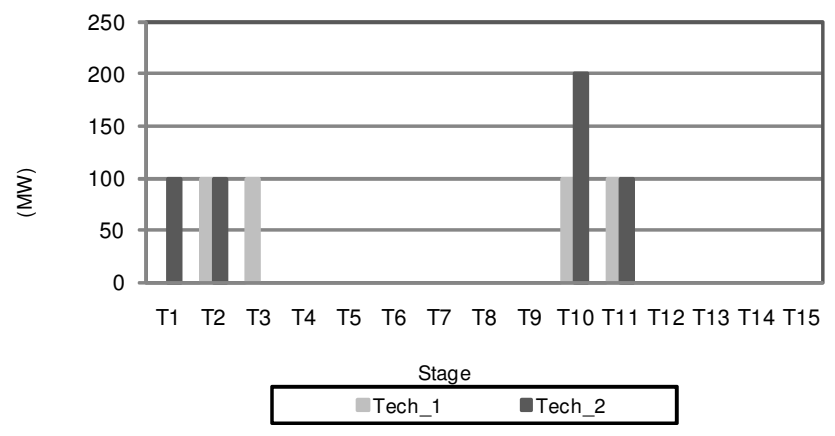

Fig. 14. New generation expansion plan obtained for Genco_1.

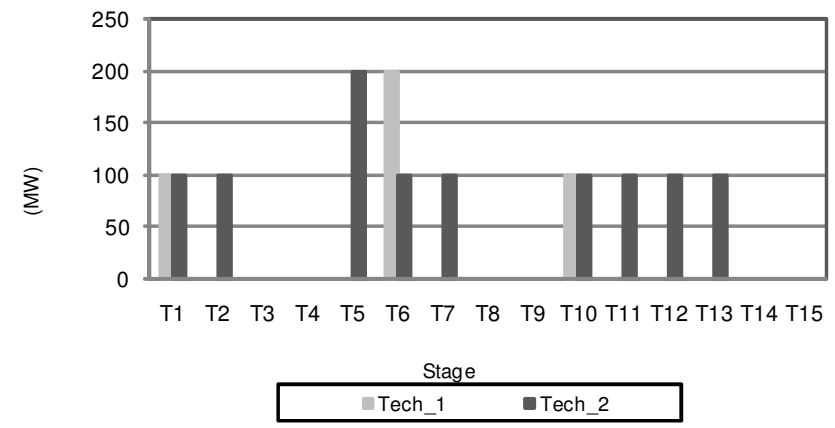

Fig. 15. New generation expansion plan obtained for Genco_2.

Comparing these plans with the ones in Figures 4 and 5, it is possible to conclude that GENCO_1 reduced the installed capacity of Tech_1 by 200 MW and increased Tech_2 by 100 MW. Regarding GENCO_2, there was a reduction of 500 MW of the installed capacity of Tech_1 and an increase of $300 \mathrm{MW}$ of Tech_2. This means that the global value of the installed capacity was reduced by $300 \mathrm{MW}$. These changes had a minor impact on the reserve margin, on LOLE and on the evolution of the electricity price. The larger change occurred in the reduction of the share of Tech_1 from $26.79 \%$, as initially reported, to $15.01 \%$ in this new situation. 


\section{CONCLUSIONS}

In this paper we described an approach to help GENCO's to prepare long-term investment plans on new generation capacity. This approach includes a dynamic model to simulate the long-term evolution of the electricity market (including the demand, the market price and the capacity factor of each technology) and the solution of individual mixed integer optimisation problems using Genetic Algorithms.

This approach can be used in a profitable way by GENCO's to help them building their plans considering the behaviour of the other competitors and also by regulatory agencies to investigate how the system will evolve along the planning period or to evaluate the impact of changes on regulatory and tariff decisions (for instance, modifying the value of feed in tariffs paid to renewables).

Another major area of application of this approach is associated with the preparation of sensitivity studies in order to evaluate the impact of changes on initial input values. On an era in which this type of studies is contaminated by uncertainties, the design of robust plans and the evaluation of their possible risk is certainly a major concern.

As a whole, this approach can help decision makers in generation companies to take more sounded decisions, namely when having more complete information and when having the possibility to investigate more completely their possible impacts.

\section{ACKNOWLEDGMENT}

The first author would like to thank Fundação para a Ciência e Tecnologia, FCT, that partially funded this research work through the PhD grant $n^{\circ}$ SFRH/BD/29243/2006.

\section{REFERENCES}

[1] B. G. Gorenstin, N. M. Campodonico, J. P. Costa, M. V. P. Pereira, "Power system expansion planning under uncertainty", IEEE Transactions on Power Systems, vol.8, no. 1, pp. 129-136, February 1993.

[2] B. F. Hobbs, "Optimization methods for electric utility resource planning", European Journal of Operational Research, vol. 83, no. 1, pp. 1 - 20, May 1995.

[3] K. Vlahos, "Capacity Planning in the Electricity Supply Industry", $\mathrm{PhD}$ Thesis, London Business School, 1990.

[4] J. Sirikum, A. Techanitisawad, V. Kachitvichyanukul, "A New Efficient GA-Benders' Decomposition Method: For Power Generation Expansion Planning with Emission Controls", IEEE Transactions on Power Systems, vol. 22, no. 3, pp. 1092 - 1100, August 2007.

[5] J. L. C. Meza, M. B. Yildrim, A. S. M. Masud, "A Model for the Multiperiod Multiobjective Power Generation Expansion Planning", IEEE Transactions on Power Systems, vol. 22, no. 2, pp. $871-878$, May 2007.

[6] C. H. Antunes, A. G. Martins, I. S. Brito, "A multiple objective mixed integer linear programming model for power generation expansion planning", Energy, vol. 29, no. 4, pp. 613 - 627, March 2004.

[7] J.-B. Park, Y.-M. Park, J.-R. Won, K. Y. Lee, "An improved genetic Algorithm for Generation Expansion Planning", IEEE Transactions on Power Systems, vol. 15, no. 3, pp. 916 - 922, August 2000.

[8] F. Wu, Z. Yen, Y. Hou, Y. Ni, "Applications of AI Techniques to Generation Planning and Investment", Proceedings of the 2004 IEEE Power Engineering Society General Meeting, Denver, USA, June 2004.

[9] J. Zhu, M. Chow, "A Review of Emerging Techniques on Generation Expansion Planning," IEEE Transactions on Power System, vol. 12, no. 4, pp. 1722-1728, November 1997.
[10] M. Yildirim, K. Erkan, S. Ozturk, "Power Generation Expansion Planning with Adaptive Simulated Annealing Genetic Algorithm", International Journal of Energy Research, vol. 30, no. 14, pp. 1188 1199, May 2006.

[11] I. Dyner, E. R. Larsen, "From Planning to Strategy in the Electricity Industry", Energy Policy, vol. 29, no. 13, pp. 1145 - 1154, November 2001.

[12] X. Zhang, "Investment in Electricity Generation and Its Determinants", Working Paper, University of Paris Sud, France, 2006.

[13] International Energy Agency, Power Generation Investment in Electricity Markets", International Energy Agency, OECD/IEA, 2003.

[14] S. Kannan, S. M. R. Slochanal, S. Baskar, P. Murugan, "Application and Comparison of Metaheuristic Techniques to Generation Expansion Planning in the Partially Deregulated Environment", IET Generation Transmission Distribution, vol. 1, no. 1, pp. 111 - 118, January 2007.

[15] J.-B. Park, J.-H. Kim, K. Y. Lee, "Generation Expansion Planning in a Competitive Environment Using a Genetic Algorithm," Proceedings of the 2002 IEEE Power Engineering Society Summer Meeting, vol. 3, pp. 1169 - 1172, July 2002.

[16] A. Botterud, M. Ilic, I. Wangensteen, "Optimal Investment in Power Generation Under Centralized and Decentralized Decision Making". IEEE Transactions on Power System, vol. 20, no 1, pp.254-263, February 2005.

[17] J. W. Forrester, "System Dynamics and the Lessons of 35 Years", in The Systemic Basics of Policy Making in the 1990s, Edt. K. B. De Greene, Sloan School of Management, MIT, USA, April 1991.

[18] A. Botterud, "Long-Term Planning in Restructured Power Systems", $\mathrm{PhD}$ Thesis, Norwegean University of Science and Technology, Trondheim, Norway, November 2003.

[19] T. Kadoya, T. Sasaki, S. Ihara, E. Larose, M. Sanford, A. K. Graham, C. A. Stephens, C. K. Eubanks, "Utilizing System Dynamics Modeling to Examine Impact of Deregulation on Generation Capacity Growth", Proceedings of the IEEE, vol. 93, no. 11, pp. 2060 - 2069, November 2005.

[20] F. Olsina, F. Garces, H.-J. Haubrich, "Modeling Long-Term Dynamics of Electricity Markets", Energy Policy, vol. 34, no. 12, pp. 1411-1433, August 2006.

[21] Powersim Software AS, "Powersim 2.5 User's Guide and Reference Manual", Powersim Press, 1996.

[22] Powersim Software AS, "Powersim Studio 7 Academic", Powersim Software AS, Bergen, Norway, 2006.

[23] A. J. C. Pereira, J. T. Saraiva, "Generation Expansion Planning in Competitive Markets", Proceedings of IEEE 2007 Power Tech Conf., Lausanne, Switzerland, July 2007.

[24] A. J. C. Pereira, J. T. Saraiva, "A Decision Support Tool for Generation Expansion Planning in Competitive Markets using System Dynamics Models", Proceedings of the IEEE 2009 PowerTech Conf., Buchareste, Romania, July 2009.

\section{BIOGRAPHIES}

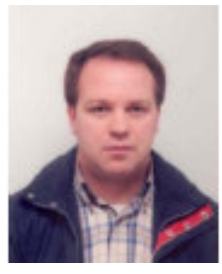

Adelino J. C. Pereira was born in Sanfins, Portugal in 1975. He received his diploma and M.Sc. degrees in Electrical Engineering and Computers from the Faculdade de Engenharia da Universidade do Porto, FEUP, Portugal, in 1998 and 2003. In 1998 he joined the Coimbra Polytechnic Institute (ISEC) where he is currently Equiparado to Adjunct Professor. $\mathrm{He}$ is a Ph.D. student at FEUP and his main research interest includes competitive markets, power systems operation and planning.

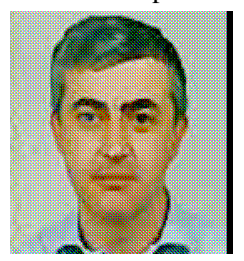

João Tomé Saraiva was born in Porto, Portugal in 1962. In 1987, 1993 and 2002 he got his MSc, PhD, and Agregado degrees in Electrical and Computer Engineering from the Faculdade de Engenharia da Universidade do Porto, FEUP, where he is currently Professor. In 1985 he joined INESC Porto - a private research institute - where he is head researcher and collaborated in several projects namely in consultancy contracts established with the Portuguese Electricity Regulatory Agency. 